\title{
LncRNA WWOX-AS1 inhibits the proliferation, migration and invasion of osteosarcoma cells
}

\author{
GANG QU $^{1 *}$, ZHIQIANG MA $^{1^{*}}$, WENXIAN TONG ${ }^{2 *}$ and JIAHUI YANG ${ }^{1}$ \\ ${ }^{1}$ Department of Osteology, The 161th Hospital of People's Liberation Army, Wuhan, Hubei 430010; \\ ${ }^{2}$ Department of Oncology, The Fifth Hospital of Wuhan, Wuhan, Hubei 430050, P.R. China
}

Received November 28, 2017; Accepted April 16, 2018

DOI: $10.3892 / \mathrm{mmr} .2018 .9058$

\begin{abstract}
Recently, numerous long non-coding (lnc)RNAs have been revealed as serving important roles in human gene regulation. Previous studies have suggested that aberrant expression of IncRNAs is associated with cancer progression and metastasis. Previous studies have also demonstrated that decreased expression of WW domain-containing oxidoreductase $(W W O X)$ is associated with poor prognosis in numerous cancer types. However, the effect of WWOX antisense RNA 1 (WWOX-AS1) in the development of cancer remains unknown. The aim of the present study was to investigate the role of WWOX-AS1 in osteosarcoma. The expression levels of WWOX-AS1 in human osteosarcoma cell lines and a normal osteoblastic cell line were investigated using reverse transcription-quantitative polymerase chain reaction (RT-qPCR). The results revealed that WWOX-AS1 expression was downregulated in osteosarcoma tissues. Furthermore, the association between WWOX-AS1 and the prognosis of patients with osteosarcoma was investigated using Kaplan-Meier and log-rank tests. The results suggested that patients exhibiting high WWOX-AS1 expression demonstrated a greater overall survival compared with patients exhibiting low WWOX-AS1 expression. In addition, overexpression and knockdown of WWOX-AS1 was performed using transfection experiments and confirmed by RT-qPCR in MG63 and SAOS2 cells, respectively. The results demonstrated that WWOX-AS1 and WWOX expression were positively correlated. Furthermore, the results of the knockdown and overexpression functional experiments suggested that WWOX-AS1 overexpression inhibited the proliferation, migration and invasion of MG63
\end{abstract}

Correspondence to: Mr. Jiahui Yang, Department of Osteology, The 161th Hospital of People's Liberation Army, 68 Huangpu Road, Wuhan, Hubei 430010, P.R. China

E-mail: jiahuiyang1127@163.com

${ }^{*}$ Contributed equally

Key words: long non-coding RNA, osteosarcoma, tumor cells, WW domain-containing oxidoreductase-antisense RNA 1, WW domain-containing oxidoreductase cells, and knockdown of WWOX-AS1 enhanced the proliferation, migration and invasion of MG63 cells in SAOS2 cells. In conclusion, the results of the present study suggested that WWOX-AS1 may represent a potential biomarker and therapeutic target for the treatment of osteosarcoma.

\section{Introduction}

Osteosarcoma is the most common primary malignant bone tumor among children and adults worldwide and is characterized by a high metastatic potential to the lung as well as poor prognosis $(1,2)$. Long non-coding $(\operatorname{lnc})$ RNAs are sequences of non-coding protein transcripts ( $>200$ nucleotides in length) with little or no protein-coding capacity (3). Unlike mRNAs, lncRNAs are associated with numerous biological and cellular processes, including the regulation of transcription and mRNA post-transcriptional levels $(4,5)$. Previous studies have revealed that lncRNAs are responsible for initiation and progression of numerous cancers due to their involvement in cellular processes (6-8). Notably, a number of functional lncRNAs have previously been revealed to be associated with osteosarcoma, including HOX transcript antisense RNA (AS) (9), breast cancer anti-estrogen resistance 4 (10), HOXA distal transcript AS (11) and urothelial cancer associated 1 (12). Numerous lncRNAs function as the antisense partners of their respective protein-coding genes, including cyclin dependent kinase inhibitor 2B-AS1 (13,14), C-terminal binding protein 1-AS (15), hepatocyte nuclear factor 1 homeobox A-AS1 (16) and proliferation arrest specific 6-AS1 (17).

LncRNA WW domain-containing oxidoreductase (WWOX)-AS1 is an antisense transcript of WWOX (18). Furthermore, $W W O X$ has been demonstrated to be strongly associated with numerous cancer types, including ovarian cancer (19), hepatocellular carcinoma (20), colon cancer (21) and bladder cancer (22). In addition, the expression levels and functions of antisense lncRNAs are frequently associated with their corresponding protein-coding genes (13-17). It has previously been demonstrated that lncRNA WWOX-AS1 is associated with ovarian cancer and that its overexpression inhibits the proliferation, migration and invasion of epithelial ovarian cancer cells (18). Furthermore, previous studies have also revealed that WWOX expression is associated with osteosarcoma and that its overexpression inhibits the progression of osteosarcoma $(23,24)$. Therefore, it may be suggested that the 
expression and function of WWOX-AS1 are associated with the progression and prognosis of osteosarcoma.

In the present study, a number of experiments were performed to investigate the functional role of WWOX-AS1 in osteosarcoma. The expression of WWOX-AS1 in osteosarcoma tissues, its association with patient prognosis and its correlation with WWOX expression levels were investigated. Furthermore, functional assays were performed via the knockdown and overexpression experiments. In conclusion, the results of the present study demonstrated the functional role of WWOX-AS1 in osteosarcoma, which may prove valuable for future osteosarcoma research.

\section{Materials and methods}

Study participants. A total of 70 patients with osteosarcoma were recruited in the present study (Table I). The 70 patients with osteosarcoma were classified into two groups: High-WWOX-AS1 group ( $\mathrm{n}=35$; WWOX-AS1 expression value $\geq$ median value) and low-WWOX-AS1 group ( $\mathrm{n}=35$; WWOX-AS1 expression value $\leq$ median value). Staging is based on Enneking system staging (25). From each patient, an osteosarcoma tissue sample and an adjacent tissue sample were collected via appropriate surgical resections. The samples were collected between March 2011 and December 2014 in the General Hospital of the People's Liberation Army (Beijing, China). Written informed consent was obtained from each patient or their guardians. The present study was approved by the Ethics Committees of the General Hospital of the People's Liberation Army.

Cancer cell lines. Three human osteosarcoma cell lines (SAOS2, MG63 and U2OS) and a normal human osteoblastic cell line (hFOB) were purchased from the American Type Culture Collection (Manassas, VA, USA). Cells were cultured in Dulbecco's Modified Eagle's Medium (DMEM; Gibco; Thermo Fisher Scientific, Inc., Waltham, MA, USA) supplemented with $10 \%$ fetal bovine serum (FBS; Invitrogen; Thermo Fisher Scientific, Inc., Waltham, MA, USA) and $1 \%$ penicillin/streptomycin solution (Invitrogen, Thermo Fisher Scientific, Inc.) at $37^{\circ} \mathrm{C}$ and $5 \% \mathrm{CO}_{2}$

RNA extraction and reverse transcription-quantitative polymerase chain reaction $(R T-q P C R)$. Total RNA was isolated from patients' tissues and cell lines using TRIzol Total RNA Reagent (Life Technologies; Thermo Fisher Scientific, Inc.) according to the manufacturer's protocol. Complementary (c) DNA was synthesized from $2 \mu \mathrm{g}$ total RNA using the RevertAid ${ }^{\mathrm{TM}}$ H Minus First Strand cDNA Synthesis kit (Takara Bio, Inc., Otsu, Japan) and cDNA was stored at $-20^{\circ} \mathrm{C}$. The RT reactions were terminated by inactivation at $95^{\circ} \mathrm{C}$ for $10 \mathrm{~min}$ and stored at $4^{\circ} \mathrm{C}$. Primers used were obtained from Chang Jing Bio-Tech, Ltd. (Changsha, China) and were identical to those used in our previously published study (18); they are presented in Table II. qPCR was performed using the SYBR PrimeScript RT-PCR kit (Takara Bio, Inc.) in an Applied Biosystems 7500 Fluorescent Quantitative PCR System (Applied Biosystems; Thermo Fisher Scientific, Inc.). The thermocycling conditions used were as follows: $95^{\circ} \mathrm{C}$ for $30 \mathrm{sec}$; followed by 40 amplification cycles at $95^{\circ} \mathrm{C}$ for $5 \mathrm{sec}$ and $60^{\circ} \mathrm{C}$ for $34 \mathrm{sec}$. The values were normalized to the internal control products of $\beta$-actin. Quantification of gene expression was performed using the $2^{-\Delta \Delta \mathrm{Cq}}$ method (26), with $\mathrm{Cq}$ representing the threshold cycle. Target gene expression per sample was determined using the following equation: Target in tumor tissue/target in non-tumorous tissue. All reactions were performed in triplicate and normalized to $\beta$-actin expression.

Knockdown of WWOX-AS1 in SAOS2 cells. Small interfering (si)RNA sequences specific to WWOX-AS1 and negative control (NC) siRNA were obtained from Chang Jing Bio-Tech, Ltd and termed WWOX-AS1-si-1 and WWOX-AS1-si-2 (NC) separately. The siRNA sequences used are also presented in Table III, and were identical to those used in our previously published study (18). A total of $2 \times 10^{3}$ SAOS2 cells were plated in 6-well plates and then separately transfected with $50 \mathrm{nmol} / 1$ WWOX-AS1-si-1 and $50 \mathrm{nmol} / 1$ WWOX-AS1-si-2 (NC) in vitro using Lipofectamine ${ }^{\circledR}$ RNAiMAX (Invitrogen; Thermo Fisher Scientific, Inc.), according to the manufacturer's protocol. The cells in the wells were exposed to the transfection mixture for $24 \mathrm{~h}$ at $37^{\circ} \mathrm{C}$ prior to the subsequent experiments.

Overexpression of WWOX-AS1 in osteosarcoma cells. WWOX-AS1 was cloned into BamHI-EcoRI sites of plasmid complementary (pc)DNA3.1 vectors (Promega Corporation, Madison, WI, USA). The MG63 cells that expressed low levels of WWOX-AS1 were transfected with 100 ng pcDNA-WWOX-AS1 or empty vector using Lipofectamine ${ }^{\circledR} 2000$ (Invitrogen; Thermo Fisher Scientific, Inc.) according to the manufacturer's protocol. The cells in the wells were exposed to the transfection mixture for $24 \mathrm{~h}$ at $37^{\circ} \mathrm{C}$ prior to the subsequent experiments.

Cell proliferation assay. Cell proliferation was investigated using a MTS (3-(4,5-dimethylthiazol-2-yl)-5-(3-carboxymethox yphenyl)-2-(4-sulfophenyl)-2H-tetrazolium) assay kit (Promega Corporation, Madison, WI, USA) in accordance with the manufacturer's protocol. MG63 and SAOS2 cells (2,000 cells/well) were separately plated in 96-well plates. MTS reagent $(20 \mu \mathrm{l})$ was added to each well containing $100 \mu \mathrm{l}$ DMEM culture medium supplemented with $10 \%$ FBS. Plates were subsequently incubated for $2 \mathrm{~h}$ at $37^{\circ} \mathrm{C}$ in a humidified atmosphere containing $5 \% \mathrm{CO}_{2}$. The absorbance was measured at a wavelength of $490 \mathrm{~nm}$ using a plate reader. The optical density (OD) values at 24, 48, 72 and $96 \mathrm{~h}$ following transfection were determined and normalized to the OD value at $0 \mathrm{~h}$. Relative cell proliferation was subsequently determined.

Scratch wound-healing assay. A total of $24 \mathrm{~h}$ post-transfection, MG63 and SAOS2 cells were seeded into a 6-well culture plate until a confluent monolayer was established and then cultured at $37^{\circ} \mathrm{C}$. Following this, uniform wounds were scraped using a sterile $200 \mu \mathrm{l}$ pipette tip and each well was washed thrice using $1 \mathrm{X}$ PBS to remove floating cells. The initial gap length $(0 \mathrm{~h})$ and the residual gap length $(48 \mathrm{~h})$ following wound initiation were calculated from images acquired using a fluorescence inversion microscope system (Nikon Corporation, Tokyo, Japan) at a magnification of $\mathrm{x} 200$.

Matrigel invasion assay. Cell invasion assays were performed using modified Boyden Chambers (BD Biosciences, Franklin Lakes, NJ, USA) according to the manufacturer's protocol. A total of $2 \times 10^{3}$ cells were suspended in $100 \mu$ l of DMEM 
Table I. Characteristics of patients with osteosarcoma participating in the present study.

\begin{tabular}{|c|c|c|c|c|}
\hline Number & $\begin{array}{c}\text { Age } \\
\text { (years) }\end{array}$ & Sex & Stage & Tumor location \\
\hline 1 & 16 & M & III A & Proximal end of tibia \\
\hline 2 & 27 & $\mathrm{~F}$ & III A & Proximal end of tibia \\
\hline 3 & 41 & $\mathrm{~F}$ & III B & Proximal end of humerus \\
\hline 4 & 40 & $\mathrm{~F}$ & I B & Proximal end of humerus \\
\hline 5 & 40 & $\mathrm{~F}$ & II A & Distal end of humerus \\
\hline 6 & 15 & $\mathrm{~F}$ & II B & Proximal end of tibia \\
\hline 7 & 19 & M & II A & Proximal end of tibia \\
\hline 8 & 42 & $\mathrm{~F}$ & II B & Proximal end of tibia \\
\hline 9 & 11 & M & II A & Proximal end of tibia \\
\hline 10 & 46 & $\mathrm{~F}$ & I A & Proximal end of femur \\
\hline 11 & 29 & M & II & Distal end of humerus \\
\hline 12 & 15 & $\mathrm{M}$ & II A & Proximal end of tibia \\
\hline 13 & 15 & M & II A & Proximal end of tibia \\
\hline 14 & 10 & M & I A & Distal end of femur \\
\hline 15 & 12 & $\mathrm{~F}$ & II B & Proximal end of tibia \\
\hline 16 & 44 & M & II B & Proximal end of ulna \\
\hline 17 & 40 & $\mathrm{~F}$ & II A & Distal end of femur \\
\hline 18 & 41 & M & I A & Proximal end of tibia \\
\hline 19 & 43 & $\mathrm{~F}$ & I B & Distal end of femur \\
\hline 20 & 19 & $\mathrm{~F}$ & II B & Proximal end of femur \\
\hline 21 & 32 & M & I A & Distal end of femur \\
\hline 22 & 40 & $\mathrm{~F}$ & II B & Distal end of femur \\
\hline 23 & 14 & $\mathrm{M}$ & II B & Proximal end of humerus \\
\hline 24 & 41 & $\mathrm{M}$ & III A & Proximal end of humerus \\
\hline 25 & 17 & M & III A & Distal end of tibia \\
\hline 26 & 12 & M & II B & Proximal end of tibia \\
\hline 27 & 42 & $\mathrm{~F}$ & I B & Proximal end of tibia \\
\hline 28 & 14 & $\mathrm{~F}$ & I B & Distal end of femur \\
\hline 29 & 21 & $\mathrm{~F}$ & I B & Distal end of femur \\
\hline 30 & 20 & $\mathrm{M}$ & II B & Proximal end of tibia \\
\hline 31 & 19 & $\mathrm{~F}$ & II B & Proximal end of femur \\
\hline 32 & 47 & $\mathrm{~F}$ & III A & Distal end of femur \\
\hline 33 & 8 & $\mathrm{M}$ & I B & Distal end of femur \\
\hline 34 & 47 & $\mathrm{M}$ & III A & Distal end of femur \\
\hline 35 & 43 & $\mathrm{~F}$ & II B & Distal end of ulna \\
\hline 36 & 15 & M & III B & Distal end of tibia \\
\hline 37 & 41 & $\mathrm{~F}$ & I A & Proximal end of humerus \\
\hline 38 & 40 & $\mathrm{~F}$ & II A & Distal end of ulna \\
\hline 39 & 41 & $\mathrm{~F}$ & I B & Distal end of ulna \\
\hline 40 & 45 & $\mathrm{~F}$ & III B & Proximal end of tibia \\
\hline 41 & 55 & M & I A & Proximal end of tibia \\
\hline 42 & 50 & $\mathrm{~F}$ & I B & Distal end of tibia \\
\hline 43 & 12 & M & III A & Distal end of femur \\
\hline 44 & 18 & $\mathrm{~F}$ & III A & Proximal end of tibia \\
\hline 45 & 15 & M & II A & Proximal end of tibia \\
\hline 46 & 16 & $\mathrm{~F}$ & I B & Distal end of femur \\
\hline 47 & 43 & $\mathrm{~F}$ & II B & Distal end of tibia \\
\hline 48 & 28 & M & II B & Distal end of tibia \\
\hline 49 & 40 & $\mathrm{~F}$ & III A & Proximal end of femur \\
\hline 50 & 10 & M & I B & Proximal end of tibia \\
\hline
\end{tabular}

Table I. Continued.

\begin{tabular}{|c|c|c|c|c|}
\hline Number & $\begin{array}{c}\text { Age } \\
\text { (years) }\end{array}$ & Sex & Stage & Tumor location \\
\hline 51 & 48 & M & II A & Proximal end of femur \\
\hline 52 & 42 & $\mathrm{~F}$ & I B & Proximal end of femur \\
\hline 53 & 18 & M & II A & Distal end of femur \\
\hline 54 & 20 & M & I A & Proximal end of femur \\
\hline 55 & 45 & $\mathrm{~F}$ & I B & Proximal end of tibia \\
\hline 56 & 45 & $\mathrm{~F}$ & II A & Distal end of femur \\
\hline 57 & 43 & $\mathrm{~F}$ & III A & Distal end of femur \\
\hline 58 & 15 & M & I A & Proximal end of tibia \\
\hline 59 & 18 & M & III A & Distal end of femur \\
\hline 60 & 42 & M & III A & Distal end of femur \\
\hline 61 & 43 & M & I B & Distal end of femur \\
\hline 62 & 16 & M & III A & Distal end of tibia \\
\hline 63 & 50 & M & I B & Distal end of femur \\
\hline 64 & 42 & $\mathrm{~F}$ & III A & Proximal end of tibia \\
\hline 65 & 15 & M & I B & Proximal end of humerus \\
\hline 66 & 15 & $\mathrm{~F}$ & I B & Distal end of femur \\
\hline 67 & 16 & $\mathrm{~F}$ & I A & Distal end of femur \\
\hline 68 & 18 & M & I B & Distal end of femur \\
\hline 69 & 49 & M & II A & Proximal end of tibia \\
\hline 70 & 11 & M & II A & Distal end of femur \\
\hline
\end{tabular}

Staging is based on Enneking system staging (26). F, female; M, male.

supplemented with $10 \%$ FBS in the lower chamber, and then plated in upper chambers with Matrigel-coated inserts (pore size, $8 \mu \mathrm{m}$ ) in 24-well culture plates (BD Biosciences). Invasive cells were stained at $42^{\circ} \mathrm{C}$ using $0.1 \%$ crystal violet solution for $15 \mathrm{~min}$ and counted whilst passing through the filter. The average invasion rate was calculated using a fluorescence inversion microscope system (Nikon Corporation) at a magnification of $x 200$; the increasing number of invasive cells as a proportion of the entire cell population after $48 \mathrm{~h}$.

Statistical analysis. Each experiment was repeated at least three times. The Student's t-test and one-way analysis of variance followed by the Newman-Keuls method were performed to analyze the statistical differences between two groups and multiple groups, respectively. The $\chi^{2}$ test was used to perform correlation analysis of clinical data. Correlation between gene expression levels was investigated using Pearson's correlation coefficient. Survival analysis was performed using the Kaplan-Meier method, and differences between the survival probabilities of each patient group were compared using a log-rank test. The values are expressed as the mean \pm standard deviation. Data analyses were performed using SPSS software (version 18.0; SPSS, Inc., Chicago, IL, USA). P $<0.05$ was considered to indicate a statistically significant difference.

\section{Results}

WWOX-AS1 is downregulated in osteosarcoma and is associated with prognosis of patients. WWOX-AS1 expression 
Table II. Primers for quantitative polymerase chain reaction analysis.

Primer sequence

\begin{tabular}{lll}
\cline { 2 - 3 } Gene name & \multicolumn{1}{c}{ Forward $\left(5^{\prime} \rightarrow 3^{\prime}\right)$} & Reverse $\left(5^{\prime} \rightarrow 3^{\prime}\right)$ \\
\hline$\beta$-actin & CCACTGGCATCGTGATGGA & CGCTCGGTGAGGATCTTCAT \\
$W W O X$ & CAAGGGCGAGTGAAGCAGT & GGCGGAGGGTGGTATTTTGT \\
WWOX-AS1 & CAGAGTGAGAACCACTGGTGAT & CGAAGGATGAGTTAAAAAGTTT
\end{tabular}

WWOX, WW domain-containing oxidoreductase; WWOX-AS1, WW domain-containing oxidoreductase-antisense RNA 1.

Table III. Sequences for small interfering RNA analysis.

\section{Sequence}

\begin{tabular}{lll}
\cline { 2 - 3 } Gene name & Sense $\left(5^{\prime} \rightarrow 3^{\prime}\right)$ & Antisense $\left(5^{\prime} \rightarrow 3^{\prime}\right)$ \\
\hline WWOX-AS1-si-1 & AUGGAUAUUUCCAAGCUGGAACCGA & UCGGUUCCAGCUUGGAAAU \\
WWOX-AS1-si-2 (NC) & AUUGAAAAUGUAAGAACUAUUCUGA & UCAGAGAUAGUUCUUACA \\
& & UUUUCAAUCA
\end{tabular}

Si, small interfering; WWOX-AS1, WW domain-containing oxidoreductase-antisense RNA 1; NC, negative control.

Table IV. Correlation between WW domain-containing oxidoreductase-antisense RNA 1 expression and clinicopathological characteristics of patients with osteosarcoma.

\begin{tabular}{|c|c|c|c|c|}
\hline Characteristics & Low WWOX-AS1 expression & High WWOX-AS1 expression & $\chi^{2}$ & P-value \\
\hline Sex & & & 0.9150 & 0.2430 \\
\hline Male & 20 & 16 & & \\
\hline Female & 15 & 19 & & \\
\hline Age (years) & & & 2.8091 & 0.0937 \\
\hline$<40$ & 22 & 15 & & \\
\hline$\geq 40$ & 13 & 20 & & \\
\hline Tumor size (cm) & & & 0.9790 & 0.4212 \\
\hline$<6$ & 20 & 24 & & \\
\hline$\geq 6$ & 15 & 11 & & \\
\hline Differentiation & & & 1.5555 & 0.3096 \\
\hline Well & 8 & 9 & & \\
\hline Moderate & 17 & 11 & & \\
\hline Poor & 10 & 15 & & \\
\hline Pulmonary metastasis & & & 5.1851 & 0.0228 \\
\hline Yes & 12 & 4 & & \\
\hline No & 23 & 31 & & \\
\hline WHO grade & & & 1.942 & 0.0121 \\
\hline I & 7 & 19 & & \\
\hline II & 17 & 10 & & \\
\hline III & 11 & 6 & & \\
\hline
\end{tabular}

WWOX-AS1, WW domain-containing oxidoreductase-antisense RNA 1; WHO, World Health Organization.

in osteosarcoma tissues was significantly downregulated in tumor tissues compared with adjacent normal tissues (Fig. 1A).
Furthermore, WWOX-AS1 expression was revealed to be lowest in patients with World Health Organization (WHO) grade III 

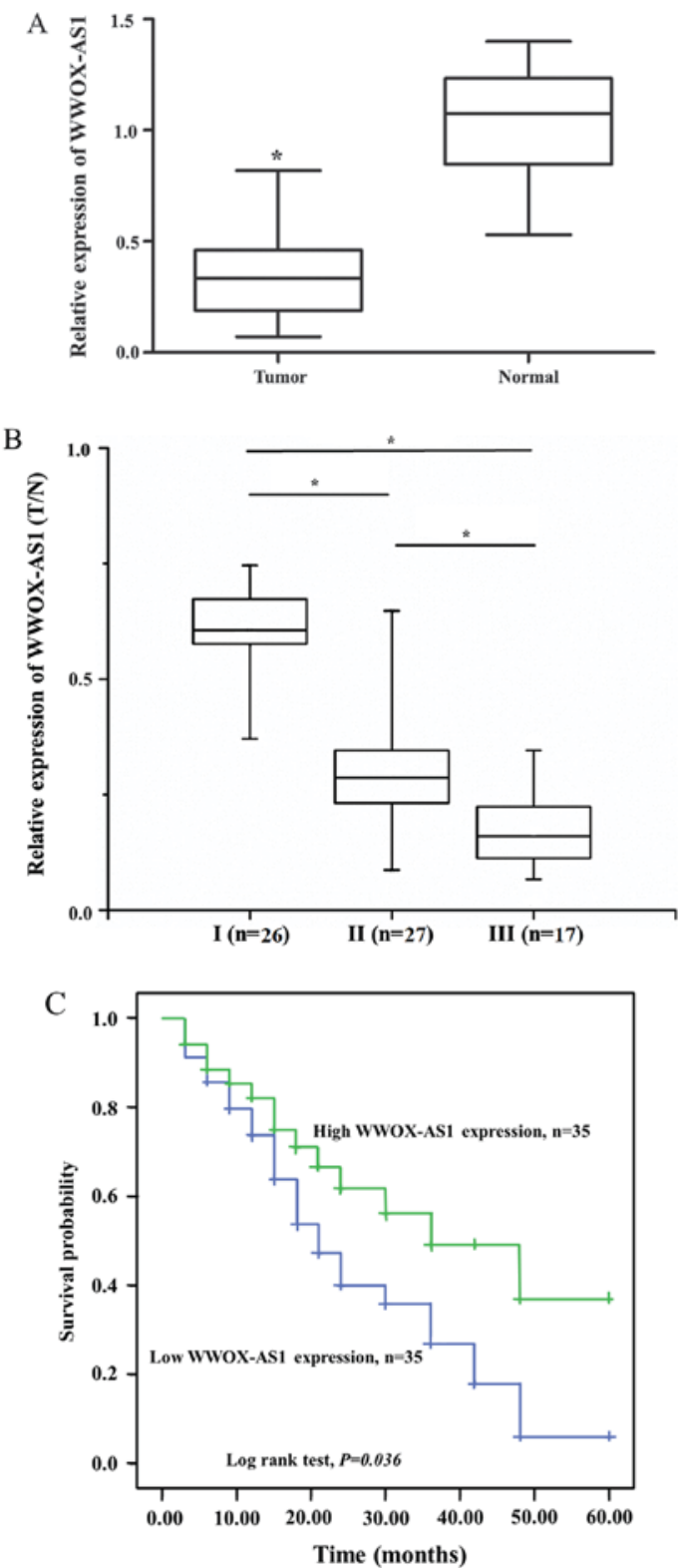

Figure 1. Relative expression of WWOX-AS1 in tissue samples obtained from 70 patients with osteosarcoma investigated using reverse transcription-polymerase chain reaction and normalized to $\beta$-actin expression. (A) WWOX-AS1 expression was significantly decreased in tumor tissues compared with normal tissues. ${ }^{\prime} \mathrm{P}<0.05$ vs. the normal group. (B) WWOX-AS1 expression was decreased in patients with WHO grade III osteosarcoma and greatest in patients with WHO grade I osteosarcoma. ${ }^{*} \mathrm{P}<0.05$. (C) Kaplan-Meier overall survival curves on the basis of WWOX-AS1 expression level. Patients with high WWOX-AS1 expression ( $\mathrm{n}=35$; WWOX-AS1 expression ratio $\geq$ median ratio) demonstrated a greater overall survival compared with patients with low WWOX-AS expression ( $\mathrm{n}=35$; WWOX-AS1 expression ratio $\leq$ median ratio; $\mathrm{P}=0.036$ ). WWOX, WW domain-containing oxidoreductase; WWOX-AS1, WWOX antisense RNA 1; WHO, World Health Organization; T/N, target in tumor tissue/target in non-tumorous tissue.

osteosarcoma, and highest in patients with WHO grade I osteosarcoma (Fig. 1B). In osteosarcoma tissue specimens, the association between WWOX-AS1 expression and the prognosis of patients with osteosarcoma was investigated using the Kaplan-Meier and log-rank tests. The 70 patients with osteosarcoma were classified into two groups: High-WWOX-AS1 group ( $\mathrm{n}=35$; WWOX-AS1 expression value $\geq$ median value) and

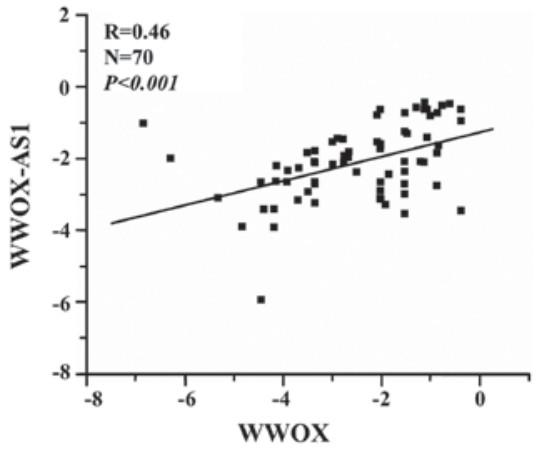

Figure 2. Correlation analysis demonstrates a positive correlation between WWOX-AS1 and WWOX expression in osteosarcoma tissue samples. WWOX, WW domain-containing oxidoreductase; WWOX-AS1, WWOX antisense RNA 1.

low-WWOX-AS1 group ( $\mathrm{n}=35$; WWOX-AS1 expression value $\leq$ median value). The results suggested that the overall survival probability of the high-WWOX-AS1 group was significantly increased compared with the low-WWOX-AS1 group (Fig. 1C; $\mathrm{P}=0.036$ ), which suggested that downregulation of WWOX-AS1 may serve an important role in the progression of osteosarcoma. As revealed in Table IV, significant differences were observed between WWOX-AS1 expression and pulmonary metastasis as well as WHO grade in the two different patient groups $(\mathrm{P}<0.05)$. However, no significant associations were revealed between WWOX-AS1 expression and sex, age, tumor size or differentiation (Table IV).

Expression of WWOX-ASI is positively correlated with WWOX expression. The association between WWOX-AS1 and WWOX was investigated using RT-qPCR. The results suggested that WWOX-AS1 and WWOX expression were positively correlated $(\mathrm{R}=0.46, \mathrm{P}<0.001$; Fig. 2).

Alterations in WWOX expression correspond with the expression of WWOX-AS1 following WWOX-AS1 overexpression or knockdown in osteosarcoma cell lines. The expression of WWOX-AS1 was investigated in three human osteosarcoma cell lines (SAOS2, MG63 and U2OS) and one human normal osteoblastic cell line (hFOB) using RT-qPCR. The results demonstrated that the expression level of WWOX-AS1 in hFOB was significantly increased compared with all three human osteosarcoma cell lines. Furthermore, SAOS2 and U2OS cells were revealed to exhibit a significantly enhanced expression of WWOX-AS1 compared with MGC3 cells (Fig. 3A).

In addition, WWOX-AS1 overexpression was analyzed using RT-qPCR. WWOX-AS1 pcDNA was constructed and transfected into MG63 cells. The results verified that WWOX-AS1 expression was significantly enhanced in the pcDNA WWOX-AS1 group compared with the pcDNA control group. In addition, the result revealed that WWOX expression was also significantly upregulated in the pcDNA WWOX-AS1 group compared with the pcDNA control group (Fig. 3B).

Furthermore, WWOX-AS1 knockdown was investigated via RT-qPCR. WWOX-AS1 siRNA was transfected into SAOS2 cells, and the results demonstrated that both WWOX-AS1 and WWOX expression levels were significantly downregulated in the WWOX-AS1 siRNA group compared with the NC 
group (Fig. 3C). Therefore, the results suggested that $W W O X$ expression may be regulated by lncRNA WWOX-AS1 in osteosarcoma.

Proliferation, migration and invasion abilities of MG63 cells are suppressed following WWOX-AS1 overexpression. To investigate the role of WWOX-AS1 in osteosarcoma, WWOX-AS1 overexpression in MG63 cells was performed. The results demonstrated that the proliferation of MG63 cells transfected with pcDNA WWOX-AS1 was significantly inhibited compared with the pcDNA control group with increased time (Fig. 4A). In addition, the results of the wound healing assay revealed that the migration of MG63 cells transfected with pcDNA WWOX-AS1 was significantly suppressed compared with the pcDNA control group (Fig. 4B). Furthermore, the results of the Matrigel invasion assay demonstrated that the invasive ability of cells transfected with pcDNA WWOX-AS1 was significantly decreased compared with the pcDNA control group (Fig. 4C). In conclusion, the results suggested that the proliferation, migration and invasion of MG63 cells were suppressed following WWOX-AS1 overexpression.

Proliferation, migration and invasion of SAOS2 cells are enhanced following WWOX-AS1 knockdown. To further investigate the role of WWOX-AS1 in osteosarcoma, WWOX-AS1 knockdown in SAOS2 cells was performed. The results demonstrated that proliferation of cells transfected with WWOX-AS1 siRNA was significantly enhanced compared with the NC group with increased time (Fig. 5A). The results of the scratch wound-healing assay revealed that cell migration in the WWOX-AS1 siRNA group was significantly enhanced compared with the NC group (Fig. 5B). In addition, the results of the Matrigel invasion assay demonstrated that cell invasion was significantly increased in the WWOX-AS1 siRNA group compared with the $\mathrm{NC}$ group (Fig. 5C). In conclusion, the results suggested that the proliferation, migration and invasion of SAOS2 cells were enhanced following WWOX-AS1 knockdown.

\section{Discussion}

Osteosarcoma is the most prevalent primary bone tumor in children, adolescents and elderly adults (1). In recent years, a number of therapeutic strategies have been used in the treatment of osteosarcoma, including surgery, multiagent chemotherapy and traditional Chinese medical treatments (27-33). However, current treatments for osteosarcoma are still limited in their effectiveness, as the molecular mechanisms underlying the pathogenesis of osteosarcoma have not been fully determined. Therefore, to improve outcomes for patients, it is important to elucidate the potential mechanism and underlying regulation process in osteosarcoma.

Previously, it has been demonstrated that antisense lncRNAs may regulate their sense transcript in a positive or negative manner (34-36). Furthermore, numerous antisense lncRNAs have been revealed to be associated with numerous malignant tumors (13-17). LncRNA WWOX-AS1 is the antisense transcript of WWOX and has been demonstrated to regulate the progression of numerous cancers, including
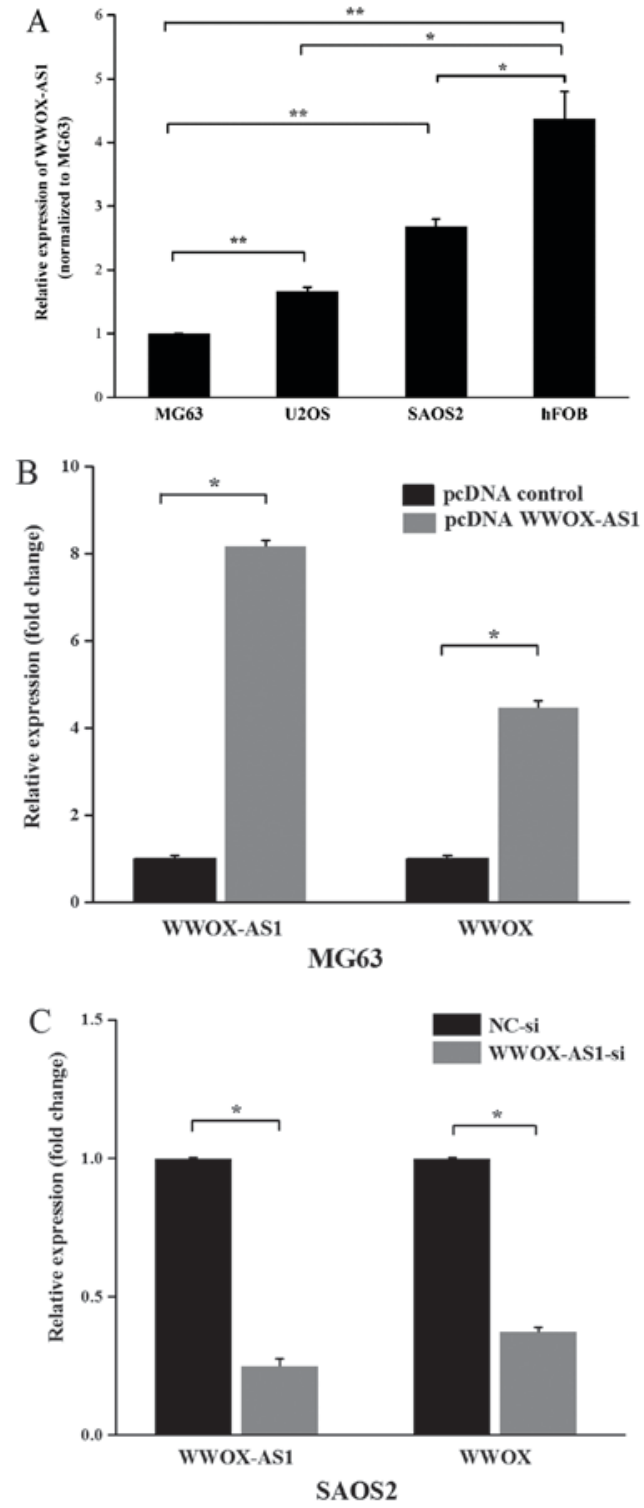

Figure 3. WWOX and WWOX-AS1 expression levels are increased in MG63 cells transfected with pcDNA WWOX-AS1 and decreased in SAOS2 cells transfected with WWOX-AS1-si. (A) Among the three human osteosarcoma cell lines investigated, the expression of WWOX-AS1 was revealed to be greatest in SAOS2 cells and lowest in MG63 cells. (B) WWOX-AS1 and WWOX expression levels were significantly upregulated in MG63 cells following transfection with pcDNA-WWOX-AS1. (C) WWOX-AS1 and WWOX expression levels were significantly decreased in SAOS2 cells following transfection with WWOX-AS1-si. ${ }^{*} \mathrm{P}<0.05$ and ${ }^{* *} \mathrm{P}<0.01$. WWOX, WW domain-containing oxidoreductase; WWOX-AS1, WWOX antisense RNA 1; NC, negative control; si, small interfering RNA; pcDNA, plasmid complementary DNA.

osteosarcoma $(23,24)$, ovarian cancer (19), hepatocellular carcinoma (20), colon cancer (21) and bladder cancer (22). However, the effect of WWOX-AS1 on the progression of osteosarcoma remains undetermined. The present study investigated the expression level of WWOX-AS1 in human tissues and osteosarcoma cell lines. The functional role of WWOX-AS1 was also analyzed in osteoblastic cell lines. The results revealed that the expression of WWOX-AS1 was downregulated in osteosarcoma tissues and cell lines compared with healthy tissues and normal human osteoblastic cells, respectively. The association between prognosis 

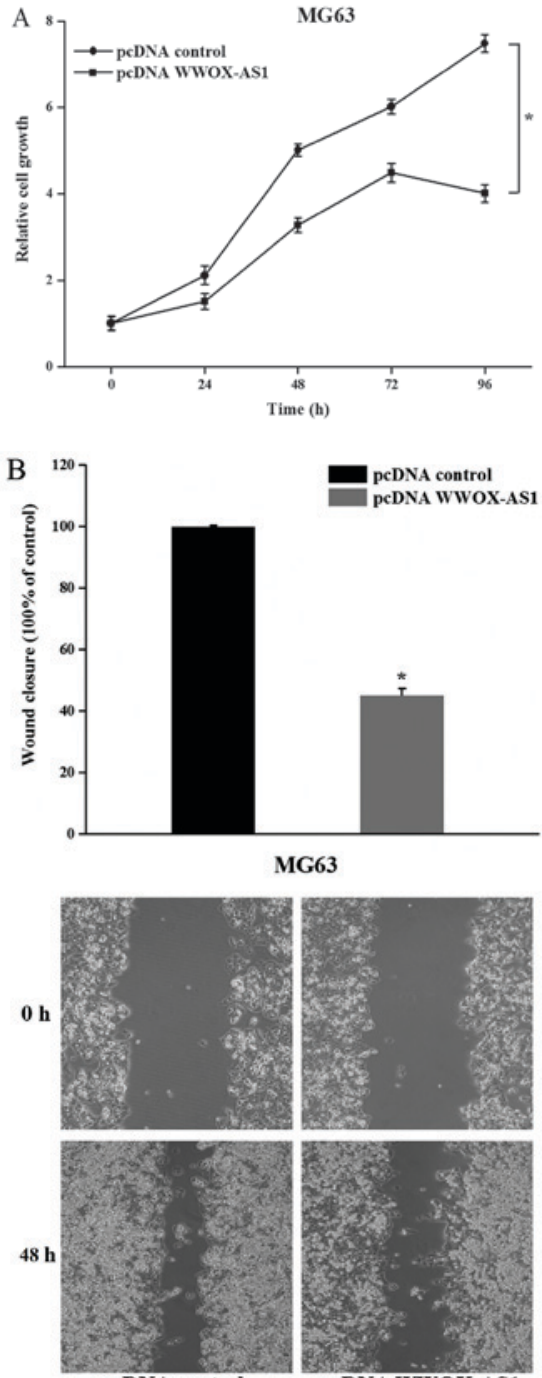

peDNA control
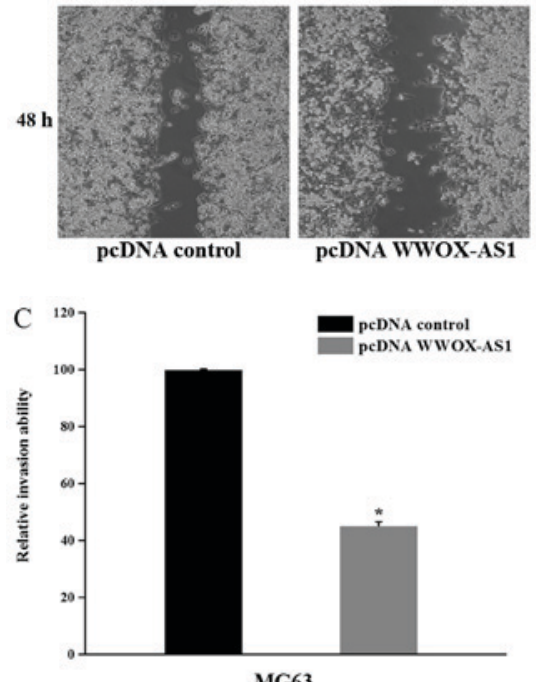

MG63

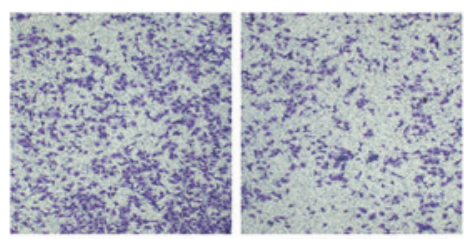

peDNA control

pcDNA WWOX-AS1

Figure 4. Proliferation, migration and invasion of MG63 cells are suppressed following transfection with pcDNA WWOX-AS1 (magnification, $\mathrm{x} 200$ ). (A) Proliferation of MG63 cells transfected pcDNA WWOX-AS1 was significantly decreased compared with the pcDNA control group. (B) Scratch wound healing assays revealed that migration of MG63 cells transfected with pcDNA WWOX-AS1 was significantly suppressed compared with the pcDNA control group. (C) Matrigel invasion assays revealed that the invasion of MG63 cells transfected with pcDNA WWOX-AS1 was significantly suppressed compared with the pcDNA control group. ${ }^{*}<0.05$ vs. the pcDNA control group. WWOX, WW domain-containing oxidoreductase; WWOX-AS1, WWOX antisense RNA 1; pcDNA, plasmid complementary DNA.
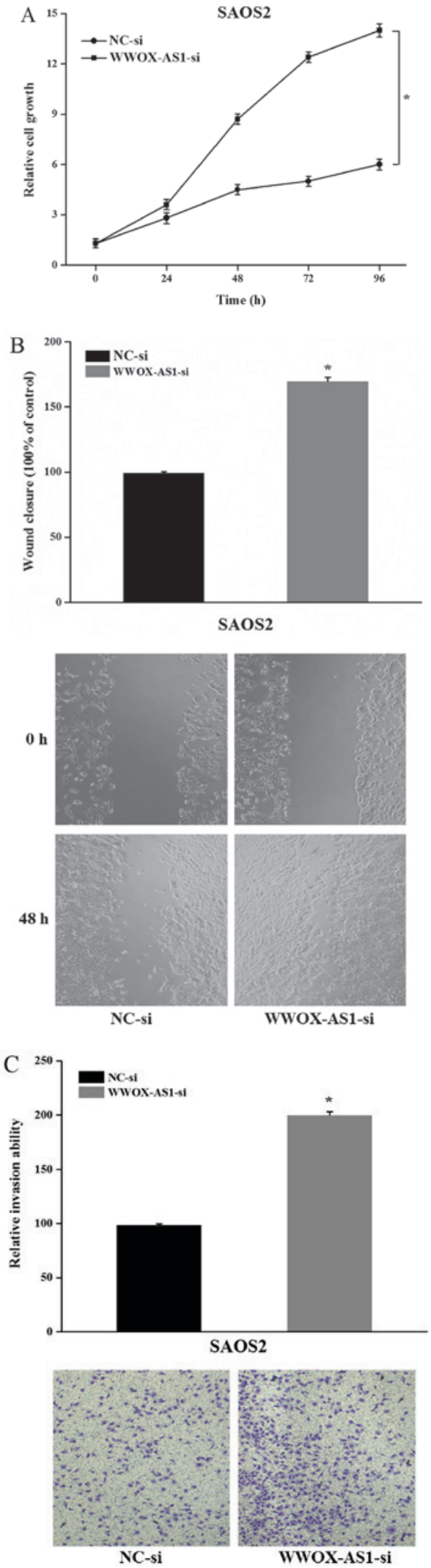

Figure 5. Proliferation, migration and invasion of SAOS2 cells are enhanced following transfection with WWOX-AS1-si (magnification, x200). (A) Proliferation of SAOS2 cells transfected with WWOX-AS1-si was significantly increased compared with the NC group. (B) Scratch wound-healing assays revealed that migration of SAOS2 cells transfected with WWOX-AS1-si was significantly enhanced compared with the NC group.(C) Matrigel invasion assays revealed that invasion of SAOS2 cells transfected with WWOX-AS1-si was significantly enhanced compared with the NC-si group. ${ }^{*} \mathrm{P}<0.05$ vs. the NC-si group. WWOX, WW domain-containing oxidoreductase; WWOX-AS1, WWOX antisense RNA 1; NC, negative control; si, small interfering RNA. 
of patients and WWOX-AS1 expression was also determined using Kaplan-Meier and log-rank tests, which suggested that patients with high WWOX-AS1 expression exhibited a greater overall survival compared with patients with low WWOX-AS1 expression. Furthermore, the results of the present study demonstrated that WWOX-AS1 and WWOX expression levels were positively correlated in patients. In addition, the expression levels of WWOX were revealed to be significantly enhanced and suppressed following WWOX-AS1 overexpression and knockdown in osteosarcoma cell lines, respectively; which further suggested that the expression levels of WWOX-AS1 and WWOX are positively correlated in osteosarcoma. These results suggested that lncRNA WWOX-AS1 may regulate $W W O X$ expression. Similar results were also demonstrated in our previous study regarding ovarian cancer (18).

To date, numerous IncRNAs have been identified as potent prognostic predictors in the progression of osteosarcoma (9-12). Therefore, the present study aimed to determine whether lncRNA WWOX-AS1 could suppress tumor progression as well as suppress the proliferation, migration and invasion of cells associated with osteosarcoma. The results demonstrated that decreased expression of WWOX-AS1 was correlated with elevated WHO grades, and that patients in the high-WWOX-AS1 group demonstrated a greater overall survival compared with patients in the low-WWOX-AS1 group. Therefore, it can be suggested that WWOX-AS1 is associated with tumor development in osteosarcoma. This may be attributed to the association between WWOX and the prognosis of osteosarcoma. For example, Zhang et al (23) demonstrated that WWOX gene polymorphisms may represent predictive factors for the assessment of risks and outcomes associated with osteosarcoma. Kurek et al (37) revealed the occurrence and frequency of $W W O X$ deletion in osteosarcoma as well as the prognostic and therapeutic significance of WWOX in osteosarcoma. The results of the present study demonstrated that WWOX-AS1 overexpression suppressed the proliferation, migration and invasion of MG63 cells. In addition, the results demonstrated that WWOX-AS1 knockdown enhanced the proliferation, migration and invasion of SAOS2 cells. These results demonstrated the inhibitory role of WWOX-AS1 on the proliferation, migration and invasion abilities of osteosarcoma cells. Previous studies have demonstrated that, as a tumor suppressor gene, WWOX could suppress the proliferation, migration, invasion and tumorigenicity of osteosarcoma cells (38), most likely via regulation of runt related transcription factor 2 (RUNX2) (24). This further suggests that there is an association between WWOX-AS1 and WWOX. In addition, genes regulated by WWOX in osteosarcoma may provide important information regarding the underlying mechanism of WWOX-AS1 in osteosarcoma. RUNX2 has been previously demonstrated to activate genes associated with angiogenesis (vascular endothelial growth factor and osteopontin) (39), metastasis and invasion [matrix metallopeptidase (MMP)2, MMP9 and MMP13] $(40,41)$, and survival (survivin) (42). It has been previously demonstrated that WWOX loss of function is associated with osteosarcoma progression by inhibition of numerous genes involved in metastasis via regulation of RUNX2 (24). Furthermore, a previous study demonstrated that investigation into the genomic function of RUNX2 in osteosarcoma cells provides insight into dysregulated molecular pathways in bone cancer (43). Therefore, it may be hypothesized that the molecular mechanisms underlying the involvement of WWOX-AS1 in osteosarcoma may be associated with RUNX2 or genes activated by RUNX2.

The present study, to the best of the authors knowledge, is the first to investigate the functional role of WWOX-AS1 in osteosarcoma. The results of the present study may provide novel insight into the roles of WWOX-AS1 in osteosarcoma, and whether WWOX-AS1 may represent a promising therapeutic target and novel biomarker for the treatment of osteosarcoma.

However, there were certain limitations to the present study. First, clinical information and samples were only collected from Chinese patients with osteosarcoma, therefore limiting the generalizability of the results. Second, in vivo experiments were performed using si-WWOX-AS1; however, it failed to achieve stable results (data not shown in this study). The silencing effect induced by exogenous delivery of synthetic siRNAs is transient and reactivation of the target gene typically occurs with a few days' interval (44). siRNA as exogenous RNA is easily degraded by nuclease in vivo and can therefore lead to unstable results. In addition, the present study did not investigate the association between WWOX-AS1 expression and apoptosis. Furthermore, the exact molecular mechanism of WWOX-AS1 was not determined in the present study. Considering these limitations, more extensive collection of comprehensive clinical information and subsequent analyses are required to further verify the results of the present study. Our future studies will aim to investigate the mechanisms underlying the association between WWOX-AS1 and osteosarcoma using viral transfection, and to establish stable cell lines. Future studies should further investigate important genes (including RUNX2) and signaling pathways associated with WWOX-AS1 in osteosarcoma.

\section{Acknowledgements}

The authors would like to thank Dr Yao (Department of Oncology) and Dr Yang (Department of Otorhinolaryngology) at the 161th Hospital of PLA (Wuhan, China) who collected the data and provided useful suggestions for the manuscript.

\section{Funding}

No funding was received.

\section{Availability of data and materials}

The datasets used and/or analyzed during the current study are available from the corresponding author on reasonable request.

\section{Authors' contributions}

GQ, ZM and WT contributed equally to this article. GQ conceived and designed the study and drafted the manuscript. ZM and WT analyzed and interpreted the data. JY put forward the concept of the study and reviewed the manuscript. All authors read and approved the final manuscript. 


\section{Ethics approval and consent to participate}

The present study was approved by the Ethics Committees of the General Hospital of the People's Liberation Army.

\section{Consent for publication}

Written informed consent was obtained from each patient or their guardians.

\section{Competing interests}

The authors declare that they have no competing interests.

\section{References}

1. McKenna WG, Barnes MM, Kinsella TJ, Rosenberg SA, Lack EE and Glatstein E: Combined modality treatment of adult soft tissue sarcomas of the head and neck. Int J Radiat Oncol Biol Phys 13: 1127-1133, 1987.

2. Chen X, Bahrami A, Pappo A, Easton J, Dalton J, Hedlund E, Ellison D, Shurtleff S, Wu G, Wei L, et al: Recurrent somatic structural variations contribute to tumorigenesis in pediatric osteosarcoma. Cell Rep 7: 104-112, 2014.

3. Spizzo R, Almeida MI, Colombatti A and Calin GA: Long non-coding RNAs and cancer: A new frontier of translational research? Oncogene 31: 4577-4587, 2012.

4. Lee JT: Epigenetic regulation by long noncoding RNAs. Science 338: 1435-1439, 2012.

5. Nagano T and Fraser P: No-nonsense functions for long noncoding RNAs. Cell 145: 178-181, 2011.

6. Tsai MC, Spitale RC and Chang HY: Long intergenic noncoding RNAs: New links in cancer progression. Cancer Res 71: 3-7, 2011.

7. Fan H, Zhu JH and Yao XQ: Knockdown of long noncoding RNA PVT1 reverses multidrug resistance in colorectal cancer cells. Mol Med Rep, 2018.

8. An Q, Zhou L and Xu N: Long noncoding RNA FOXD2-AS1 accelerates the gemcitabine-resistance of bladder cancer by sponging miR-143. Biomed Pharmacother 103: 415-420, 2018.

9. Zhou Q, Chen F, Fei Z, Zhao J, Liang Y, Pan W, Liu X and Zheng D: Genetic variants of lncRNA HOTAIR contribute to the risk of osteosarcoma. Oncotarget 7: 19928-19934, 2016.

10. Chen F, Mo J and Zhang L: Long noncoding RNA BCAR4 promotes osteosarcoma progression through activating GLI2-dependent gene transcription. Tumour Biol 37: 13403-13412, 2016.

11. Li Z, Zhao L and Wang Q: Overexpression of long non-coding RNA HOTTIP increases chemoresistance of osteosarcoma cell by activating the Wnt/ $\beta$-catenin pathway. Am J Transl Res 8 : 2385-2393, 2016.

12. Li W, Xie P and Ruan WH: Overexpression of lncRNA UCA1 promotes osteosarcoma progression and correlates with poor prognosis. J Bone Oncol 5: 80-85, 2016.

13. Pasmant E, Laurendeau I, Heron D, Vidaud M, Vidaud D and Bieche I: Characterization of a germ-line deletion, including the entire INK4/ARF locus, in a melanoma-neural system tumor family: Identification of ANRIL, an antisense noncoding RNA whose expression coclusters with ARF. Cancer Res 67: 3963-3969, 2007.

14. Yu W, Gius D, Onyango P, Muldoon-Jacobs K, Karp J,Feinberg AP and Cui H: Epigenetic silencing of tumour suppressor gene p15 by its antisense RNA. Nature 451: 202-206, 2008.

15. Takayama K, Horie-Inoue K, Katayama S, Suzuki T, Tsutsumi S, Ikeda K, Urano T, Fujimura T, Takagi K, Takahashi S, et al: Androgen-responsive long noncoding RNA CTBP1-AS promotes prostate cancer. EMBO J 32: 1665-1680, 2013.

16. Yang X, Song JH, Cheng Y, Wu W, Bhagat T, Yu Y, Abraham JM, Ibrahim S, Ravich W, Roland BC, et al: Long non-coding RNA HNF1A-AS1 regulates proliferation and migration in oesophageal adenocarcinoma cells. Gut 63: 881-890, 2014.

17. Han L, Kong R, Yin DD, Zhang EB, Xu TP, De W and Shu YQ: Low expression of long noncoding RNA GAS6-AS1 predicts a poor prognosis in patients with NSCLC. Med Oncol 30: 694, 2013.
18. Tong W, Yang L, Yu Q, Yao J and He A: A new tumor suppressor lncRNA RP11-190D6.2 inhibits the proliferation, migration, and invasion of epithelial ovarian cancer cells. Onco Targets Ther 10: 1227-1235, 2017.

19. Yan H, Tong J, Lin X, Han Q and Huang H: Effect of the WWOX gene on the regulation of the cell cycle and apoptosis in human ovarian cancer stem cells. Mol Med Rep 12: 1783-1788, 2015.

20. Xie B, Zen Q, Wang X, He X, Xie Y, Zhang Z and Li H: ACK1 promotes hepatocellular carcinoma progression via downregulating WWOX and activating AKT signaling. Int J Oncol 46: 2057-2066, 2015.

21. Nowakowska M, Pospiech K, Lewandowska U, Piastowska-Ciesielska AW and Bednarek AK: Diverse effect of WWOX overexpression in HT29 and SW480 colon cancer cell lines. Tumour Biol 35: 9291-9301, 2014.

22. Li G, Sun L, Mu Z, Huang Y, Fu C and Hu B: Ectopic WWOX expression inhibits growth of 5637 bladder cancer cell in vitro and in vivo. Cell Biochem Biophys 73: 417-425, 2015.

23. Zhang N, Jiang Z, Ren W, Yuan L and Zhu Y: Association of polymorphisms in WWOX gene with risk and outcome of osteosarcoma in a sample of the young Chinese population. Onco Targets Ther 9: 807-813, 2016.

24. Del Mare S and Aqeilan RI: Tumor Suppressor WWOX inhibits osteosarcoma metastasis by modulating RUNX2 function. Sci Rep 5: 12959, 2015.

25. Jawad MU and Scully SP: In brief: Classifications in brief: Enneking classification: Benign and malignant tumors of the musculoskeletal system. Clin Orthop Relat Res 468: 2000-2002, 2010.

26. Livak KJ and Schmittgen TD: Analysis of relative gene expression data using real-time quantitative PCR and the 2(-Delta Delta C(T)) method. Methods 25: 402-408, 2001.

27. Isakoff MS, Bielack SS, Meltzer P and Gorlick R: Osteosarcoma: Current treatment and a collaborative pathway to success. J Clin Oncol 33: 3029-3035, 2015.

28. Zhang T, Li J, Yin F, Lin B, Wang Z, Xu J, Wang H, Zuo D, Wang G, Hua $Y$ and Cai Z: Toosendanin demonstrates promising antitumor efficacy in osteosarcoma by targeting STAT3. Oncogene 36: 6627-6639, 2017.

29. Liu X, Xiu LJ, Jiao JP, Zhao J, Zhao Y, Lu Y, Shi J, Li YJ, Ye M, Gu YF, et al: Traditional Chinese medicine integrated with chemotherapy for stage IV non-surgical gastric cancer: A retrospective clinical analysis. J Integr Med 15: 469-475, 2017.

30. Mondal J, Das J, Shah R and Khuda-Bukhsh AR: A homeopathic nosode, Hepatitis C 30 demonstrates anticancer effect against liver cancer cells in vitro by modulating telomerase and topoisomerase II activities as also by promoting apoptosis via intrinsic mitochondrial pathway. J Integr Med 14: 209-218, 2016.

31. Adaramoye O, Erguen B, Oyebode O, Nitzsche B, Höpfner M, Jung $\mathrm{K}$ and Rabien A: Antioxidant, antiangiogenic and antiproliferative activities of root methanol extract of Calliandra portoricensis in human prostate cancer cells. J Integr Med 13: 185-193, 2015.

32. Wang X, Wang N, Cheung F, Lao L, Li C and Feng Y: Chinese medicines for prevention and treatment of human hepatocellular carcinoma: Current progress on pharmacological actions and mechanisms. J Integr Med 13: 142-164, 2015.

33. Zang QQ, Zhang L, Gao N and Huang C: Ophiopogonin D inhibits cell proliferation, causes cell cycle arrest at $\mathrm{G} 2 / \mathrm{M}$, and induces apoptosis in human breast carcinoma MCF-7 cells. J Integr Med 14: 51-59, 2016.

34. Wahlestedt C: Natural antisense and noncoding RNA transcripts as potential drug targets. Drug Discov Today 11: 503-508, 2006.

35. Faghihi MA and Wahlestedt C: Regulatory roles of natural antisense transcripts. Nat Rev Mol Cell Biol 10: 637-643, 2009.

36. Yelin R, Dahary D, Sorek R, Levanon EY, Goldstein O, Shoshan A, Diber A, Biton S, Tamir Y, Khosravi R, et al: Widespread occurrence of antisense transcription in the human genome. Nat Biotechnol 21: 379-386, 2003.

37. Kurek KC, Del Mare S, Salah Z, Abdeen S, Sadiq H, Lee SH, Gaudio E, Zanesi N, Jones KB, DeYoung B, et al: Frequent attenuation of the WWOX tumor suppressor in osteosarcoma is associated with increased tumorigenicity and aberrant RUNX2 expression. Cancer Res 70: 5577-5586, 2010.

38. Del Mare S, Kurek KC, Stein GS, Lian JB and Aqeilan RI: Role of the WWOX tumor suppressor gene in bone homeostasis and the pathogenesis of osteosarcoma. Am J Cancer Res 1: 585-594, 2011. 
39. Zelzer E, Glotzer DJ, Hartmann C, Thomas D, Fukai N, Soker S and Olsen BR: Tissue specific regulation of VEGF expression during bone development requires Cbfa1/Runx2. Mech Dev 106: 97-106, 2001.

40. Pratap J, Javed A, Languino LR, van Wijnen AJ, Stein JL, Stein GS and Lian JB: The Runx2 osteogenic transcription factor regulates matrix metalloproteinase 9 in bone metastatic cancer cells and controls cell invasion. Mol Cell Biol 25, 8581-8591, 2005.

41. Wang X, Manner PA, Horner A, Shum L, Tuan RS and Nuckolls GH: Regulation of MMP-13 expression by RUNX2 and FGF2 in osteoarthritic cartilage. Osteoarthritis Cartilage 12: 963-973, 2004

42. Lim M, Zhong C, Yang S, Bell AM, Cohen MB and Roy-Burman P: Runx2 regulates survivin expression in prostate cancer cells. Lab Invest 90: 222-233, 2010.
43. van der Deen M, Akech J, Lapointe D, Gupta S, Young DW, Montecino MA, Galindo M, Lian JB, Stein JL, Stein GS and van Wijnen AJ: Genomic promoter occupancy of runt-related transcription factor RUNX2 in osteosarcoma cells identifies genes involved in cell adhesion and motility. J Biol Chem 287: 4503-4517, 2012

44. Elbashir SM, Harborth J, Weber K and Tuschl T: Analysis of gene function in somatic mammalian cells using small interfering RNAs. Methods 26: 199-213, 2002.

(i) (3) This work is licensed under a Creative Commons

cc) International (CC BY-NC-ND 4.0) License. 